\title{
Correction to: Editorial
}

\author{
Shuzhi Sam $\mathrm{Ge}^{1,2,3} \cdot$ Agnieszka Wykowska $^{4}$. Oussama Khatib ${ }^{5}$
}

(c) Springer Nature B.V. 2019

\section{Correction to: International Journal of Social Robotics https://doi.org/10.1007/s12369-019-00567-2}

This is to inform you that the following sentence was inadvertently omitted from the final version of https://doi.org/10. 1007/s12369-019-00567-2

The first sentence of the editorial should have read:

"We are extremely pleased to announce that our journal is celebrating its 10th anniversary! It's been now 10 years of exciting research on social robots, building a community and a vision that will last for many years ahead. Now we are entering the age of adolescence and we hope that it will smoothly bring us to maturity. Thank you to all contributors to our journal for making it grow, we are looking forward to the next decade!"

Publisher's Note Springer Nature remains neutral with regard to jurisdictional claims in published maps and institutional affiliations.
The original article can be found online at https://doi.org/10.1007/ s12369-019-00567-2.

\section{Shuzhi Sam Ge}

samge@nus.edu.sg

Agnieszka Wykowska

agnieszka.wykowska@iit.it

1 Social Robotics Laboratory, Smart Systems Institute, Department of Electrical and Computer Engineering, The National University of Singapore, Singapore, Singapore

2 Institute for Future (IFF), Qingdao University, Qingdao 266071, China

3 Centre for Robotics, UESTC, Chengdu, China

4 Social Cognition in Human-Robot Interaction, Istituto Italiano di Tecnologia, Genoa, Italy

5 Artificial Intelligence Laboratory, Department of Computer Science, Stanford University, Stanford, CA, USA 